\title{
A Camera System for Detecting Dust and Other Deposits on Solar Panels
}

\author{
E. A. Yfantis ${ }^{1}$ and A. Fayed ${ }^{2}$ \\ ${ }^{1}$ Computer Science Department, University of Nevada, Las Vegas, Nevada, USA \\ ${ }^{2}$ Mechanical Engineering Department, University of Nevada, Las Vegas, Nevada, USA \\ yfantis@cs.unlv.edu
}

\begin{abstract}
Solar panels over time, due to winds, sandstorms, bird droppings, suffer from dust, and other deposits. As a result of these deposits the sunlight is refracted, and only part of the sunlight reaches the chips inside the glass cage that are generating the electricity. In a remote area with thousands of solar panels, it is both expensive and cumbersome to send maintenance people to inspect each panel and clean it if needed. We have smart cameras with $R, G, B$, and infrared for night vision, that take the picture of each panel continuously. The picture becomes input to our classification algorithm that decides real time if the panel needs cleaning or not. Our classification algorithm consists of: our classification vector, the metric used, the training of the classifier, the testing of the classifier, and the classifier put into play for everyday use. At the present time we use a commercial camera transmitting JPEG frames wireless to our server where the classification and storage takes place. But in the near future our classification algorithm will reside on a flash memory which will be part of a circuit board that we are designing. The algorithm operates on the incoming data and will be executed by an ARM processor which will also be on the board. The circuit board also will include a CCD and Infrared camera. The hardware and software on this electronic board will be designed and programmed by the authors. Once our intelligent system detects that the panel needs cleaning it will automatically trigger a mechanism which will clean the panel.
\end{abstract}

Keywords-Multivariate distribution; Mahalanobis distance; Hotelling's T-square; misclassification.

\section{Introduction}

In a large scale solar energy array of panels build by a variety of solar panel manufacturers, each panel having a different area, and based on different type of chip architectures, the energy output is not uniform and there could be a considerable panel to panel variation. The energy output of each panel depends on the time of the day, day of the month, month of the year, year in the solar cycle, and of course the climatological conditions during the time of the day that energy measurements are obtained. In arid climates it rains very rarely so the ground is dry therefore when the wind blows the loose ground is carried by the wind and dust storms are very common. As the dust settles on the glass of the various panels the energy output falls because the dust prevents part of the light incident to the glass to enter and reach the chips generating the energy. Thus part of the light is refracted and less light goes through the glass and into chip structure designed to convert the solar energy to electricity. The loss of light energy depends on the amount of dust, the size of particulates, and the chemical composition of dust. Loss of solar energy is not just due to dust but also due to bird droppings and other factors that are yet to be explained. In arid climates there are not as many trees 
E. A. Yfantis \& A. Fayed; A Camera System For Detecting Dust And Other Deposits On Solar Panels, Advances in Image and Video Processing, Volume 2 No 5, Oct (2014); pp: 1-10

as in climates with normal amount of rain. As the birds migrate from north to warmer climates during the fall, or from warm to cooler climates during the spring, solar farms are used by the migrating birds as rest areas, and therefore get many bird dropping that reduce the electric energy output. Also during the regular season solar panels get a fair amount of bird droppings. In arid climates that often times the summers are too hot, and the winters nights are cold, solar panels provide shade in the summer and protection from the cold and the wind in the winter. Thus many small animals find rescue from the difficult climate conditions and make home under the panels. Often times some of these animals try to eat the cables attached to the panels and in the process damage the cable to the point that stops the flow of electricity from the panel to the destination. In addition to the animals and birds, the protections the panels provide from the harsh weather conditions promote the growth of various desert plants that grow faster in the shaded ground underneath the panels. Often times these plants and trees grow enough to crack the base of the panel, or lift the panel so that the orientation of the panel with respect to the sun is not what it should be, therefore the amount of light going through the glass and into the chip structure designed to convert the solar energy to electricity is less than the optimal amount. Often times these plants as they grow into trees they lift the panel and severely damage it as well as other neighboring panels that this panel falls on the top of. In addition to the above possibilities for damaging the panels and reducing the electric energy output there are others, such as vandalism, earthquakes, theft, and a number of catastrophes considered to be acts of God. For now the only purpose of our smart camera is to detect if a panel needs cleaning by applying our classifier and then if it does need cleaning to trigger a mechanism to clean the panel. As we looked at a number of cleaning mechanisms including a container with water and two sponges one to wash the panel with the water and the other to dry, or a container with a cleaning fluid and two sponges, or a mechanism consisting of small spike-like needles to crate static electricity on the dirt of the panel and attract the dirt into a vacuum, we concluded that the later is the best method and has many advantages over the previous mentioned methods. Some of the advantages of the static electricity mechanism with the vacuum are its very low maintenance. The mechanism with the water and the sponges need enormous amount of maintenance in an arid climate. The sponges dry out relatively fast in the extreme climate conditions of the summer months that the temperature could rise well over 110 degrees Fahrenheit and could need to be replaced very often adding to the expense of maintenance of the solar panel site. Furthermore water is very scarce, and evaporates very fast as it is poured on the top of the panels. As the mechanism cleans the panel with water is very possible that it would create uneven cleaning and/or leave a film of material that acts is inhibitors for the panel to utilize the solar energy in an optimal way. Replacing the water in the containers of each solar panel could be expensive and challenging. It might have an initial expense to bring the water pipe into the site and then a continuous expense to maintain the containers and their sensors that allow the proper release of the water. If a cleaning fluid is used the challenges are similar to those of using the water, but the expense could be much higher, and that includes cleaning fluid replacement, sponge and/or wiper replacement, and man power needed for the proper maintenance. The static electricity mechanism to remove the dust is very light, gets a small amount of energy from the panel itself , is relatively simple to build, therefore could be very reliable, and needed very little maintenance. In this research paper we are focusing only on building a classifier to detect if the panel needs to be cleaned or not. The steps for building our classifier included sampling, deciding which parameters our classification vector should include, train the classifier, compute the probability of misclassification which includes the probability of the classifier deciding that the panel is dirty 
although it is not, or the panel is clean although it is not. After testing the classifier we put it into play on a real solar panel site. For now the classifier is computer software on the server where the camera transmits the video of the solar panels. In the near future the classifier will be embedded into a hardware board, which will include the visual camera chip, the infrared camera, and the alarm events that will be transmitted wirelessly and alarming the care takers of the site about broken glass, a malfunction in one of the panels, cut cables, or vandalisms. Our classifier is based on the multivariate probability distribution function of the mode of the red channel, the mode of the green channel, and the mode of the blue channel. It also includes the marginal distribution function of the modes of the red, green, and blue channels. In clean panels the three dimensional vector of the mean vector of the modes has relatively low values, while as the panel gets dirty by dust particulates and bird droppings, the means of the mode vectors of the red, green, and blue channels increase. A Hotelling's T-square test shows that the mean vectors of the modes between clean and dirty panels are significantly different. Also the Mahalanobis distance is capable of classifying correctly with high probability that a panel belongs to the clean or dirty category of panels. This paper is structured as follows: First is the abstract, section I is the Introduction, section II is the Background Information, Section III is the Classification Algorithm, Section IV is the Conclusions, Section V is the References.

\section{Background Information}

\subsection{The color of the Sky}

The sky color variations from shades of blue to shades of gray indicate higher levels of concentration of particulates larger than the clean air. The clean air is composed approximately of $78.084 \%$ Nitrogen, $20.94 \%$ Oxygen, $0.934 \%$ Argon, and $0.0350 \%$ other gases [1], [2]. The color of the sky is explained by the Mie solution to Maxwell's equation, and Rayleigh's scatter approximation theory. According to Rayleigh's scatter theory the small molecule size of Nitrogen and Oxygen molecules scatter the components of the light with shorter wavelength range and thus higher frequency. These light components in the visible spectrum are the blue and violet colors. The scattering of the blue colors therefore by Nitrogen and Oxygen explains the blue color of the sky. Larger molecules scatter color components with longer wavelength. In the visible light the red color component of the white light has the larger wavelength, then the green color component has lower wavelength than the red, and the blue color component has lower wavelength than the green. When the concentration of larger molecules such as pollutants increase, the scattering of colors with longer wavelength increases. The mix of the longer wavelength color components in the atmosphere with the blue, change the color of the sky, making it more grey, or light grey. Thus when there is high concentration of water vapor molecules that cling together in that part of the sky make it look white or gray. This is the reason that clouds look white or gray. When pollution levels increase, since their molecule size is usually relatively large, that triggers the scattering of higher wavelength color components. The color of the sky is a good indicator of how clean the air is. Lighter grey colors signify the presence of water vapor, sulfur, aerosols, and soot in the atmosphere. In any given direction skylight is light scattered by all the molecules and particles in its path from the sun which is the source of daylight in the Earth. Since the atmosphere on the Earth is composed mostly of Nitrogen and Oxygen molecules, then these are the most frequent molecules that light will encounter in its trajectory. Electromagnetic waves with shorter wavelengths are affected by particles much smaller than their wavelength, scattering these wave in different directions. Waves with larger wavelength are not being affected by these small particles. As a result the blue and violet waves of the white light are scattered while traveling in the clean atmosphere turning the sky blue [3],[4],[5]. The lower intensity of the violet color compared to other colors in the light coming from the sun, combined with the 
E. A. Yfantis \& A. Fayed; A Camera System For Detecting Dust And Other Deposits On Solar Panels, Advances in Image and Video Processing, Volume 2 No 5, Oct (2014); pp: 1-10

lower sensitivity of this color by the human eyes is the main reason why the sky is blue and not violet even though the violet wavelength is smaller than the blue and scatters even more. So there is very little scattering of the red color due to its relatively large wavelength. The red color travels straight thru the atmosphere. Similarly there is very little scattering for the green color because although its wavelength is smaller than that of red is still relatively large compared to the size of Nitrogen and Oxygen. So the green light travels straight thru a clean atmosphere.

\subsection{Light and Electric Energy}

The infrared and red wavelengths having the larger wavelengths compared to the other components of the white light incident to the glass of the solar panel penetrates the glass and produces the most amount of electricity. The blue and violet wavelengths suffer the most absorption by the glass of the panel and contribute very little in the production of electric energy. As we pointed out in our previous section when the sky is cloudy due to water vapor, the red and green components of the light suffer a great deal of scattering and do not go straight thru the atmosphere. The infrared wavelengths are the ones that produce more energy following by the red, green, and blue. The energy produced by the red and green wavelengths is substantially reduced during cloudy days. Also when dust particulates, or other pollutants are in the atmosphere they cause the scattering of the larger wavelength components of light thus the energy produced by the solar panels is reduced. Dust storms are very common in arid climates, especially during the summer months when the ground is very dry and the top soil is sandy. Sandstorms are responsible for depositing dust and sand on the solar panels. As the sunlight passes thru these particulates the larger wavelengths that normally are the larger energy producers, scatter, and as a result of this scattering less light energy is reaching the chips producing the energy and therefore less energy is produced. Birds flying over the solar panel area, or stopping on solar panels for a rest or out of curiosity, often times they deposit their droppings. Bird droppings usually form a thick coating on an area of the panel preventing the sun light in that area from going thru. It is very difficult for one to change the weather or the pollution in the air. It is not difficult to detect the dust and sand on the glass and clean it. As we mentioned before, our approach is not to use water or other liquid for cleaning, but to use a small vacuum cleaner flying over the panel in a methodical way that goes thru in a raster type of way and visits every point of the panel from top to button twice. During the first pass it vacuums all the gravel, dust, and droppings it can. During The second pass it activates the needles that have static current, thus charging the dust and droppings on the glass with opposite static current. Thus our system visits every point on the glass in a raster scanning fashion, the needles attract the remaining deposits on the glass, and those deposits go thru the vacuum and get deposited thru a pipe system on the ground by the base of panel. This is a relatively simple system, draws the energy from the panel itself, does a very good job in cleaning, does not leave any film on the glass, has a relatively small manufacturing and installation cost, it needs very little maintenance. It is very easy to train a technician to repair or replace the system.

\section{Classification Algorithm}

As we examine an image of a clean panel (Fig. 1), and compute the probability density functions of the red channel, the green channel, and the blue channel of the pixels of the image (Fig. 3) we observe that each one of the marginal probability density functions for each one of the channels is skewed to the left with a maximum (mode) to the left of the 127.5 value. Here we assume that the scale of the red channel of the pixels is between $0-255$, similarly the scales of the green and blue channels of the pixels are also between $0-255$. Thus in the graphs of the probability density function 
of the color channels the scale on the $x$-axis is between 0-255. Fig. 3(a) gives the univariate probability distribution functions of the red, green, and blue, channels, for a clean panel. The mode for the red channel is 56 , for the green is 60 , and for the blue is 84 . The $x$-axis for each one of the channels is from 0-255. Fig. 3(b) gives the univariate probability distribution functions of the red, green, and blue channels, for a dusted panel. The mode for the red channel is 79 , for the green is 82 , and for the blue is 105 . Based on a sample of 50 pictures of dirty panels and fifty of clean ones, the gamma distribution provides enough flexibility to model these probability functions. The gamma density function is of the form [6], [7]:

$$
f(x)=\frac{x^{\alpha-1} e^{-\frac{x}{\beta}}}{\Gamma(\alpha) \beta^{\alpha}}, x>0, \alpha, \beta>0
$$

Theorem 1, gives an estimate of the global mode of the gamma density function in terms of its parameters $\alpha, \beta$, and also estimates the parameters from the data.

Theorem 1. The global mode of the gamma density function (the point $x$ that maximizes the density function $\mathrm{f}(\mathrm{x})$ is $x_{m}=(\alpha-1) \beta$, if $\hat{x}$ is the global mode based on the empirical density function computed from the data, and $\bar{x}$ is the average then an estimate of the parameters $\alpha, \beta$, from the data is: $\widehat{\alpha}=\frac{\bar{x}}{\bar{x}-\hat{x}}$ and $\widehat{\beta}=\bar{x}-\hat{x}$

\section{Proof}

The global mode $x_{m}$ of the gamma probability function is the point that maximizes the density function. Namely the point for which the first derivative of the function $f(x)$ is zero and the second derivative is negative.

$$
f^{\prime}(x)=e^{-\frac{x}{\beta}} \frac{(\alpha-1) x^{\alpha-2}-\frac{x^{\alpha-1}}{\beta}}{\Gamma(\alpha) \beta^{\alpha}}=0
$$

The solution to the above equation is

$$
\begin{gathered}
x_{m}=(\alpha-1) \beta \\
f^{\prime \prime}(x)=-\frac{1}{\beta} e^{-\frac{x}{\beta} \frac{(\alpha-1) x^{\alpha-2}-\frac{x^{\alpha-1}}{\beta}}{\Gamma(\alpha) \beta^{\alpha}}}+e^{-\frac{x}{\beta} \frac{(\alpha-1)(\alpha-2) x^{\alpha-3}-\frac{(\alpha-1) x^{\alpha-2}}{\beta}}{\Gamma(\alpha) \beta^{\alpha}}}
\end{gathered}
$$

For the type of gamma probability function that fits this data $\alpha>2$ always. Thus

$$
f^{\prime \prime}\left(x_{m}\right)=-e^{-(\alpha-1)} \frac{((\alpha-1) \beta)^{\alpha-3}}{\Gamma(\alpha) \beta^{\alpha}}<0
$$

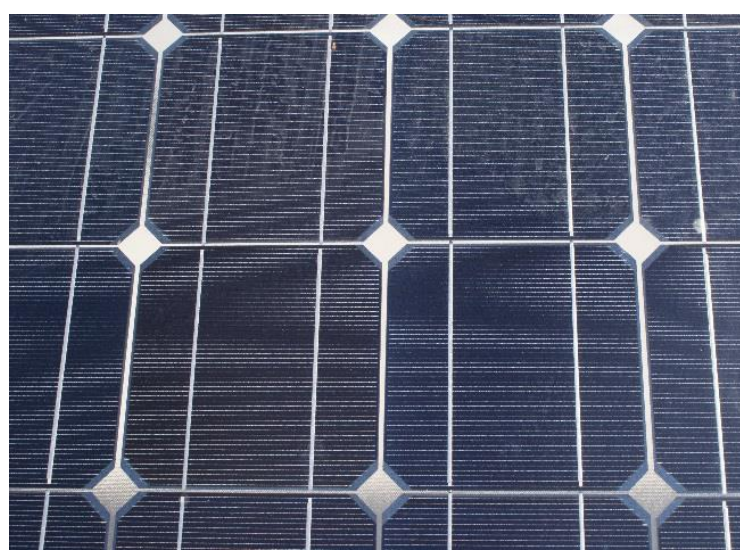

Figure 1: Image of a clean panel

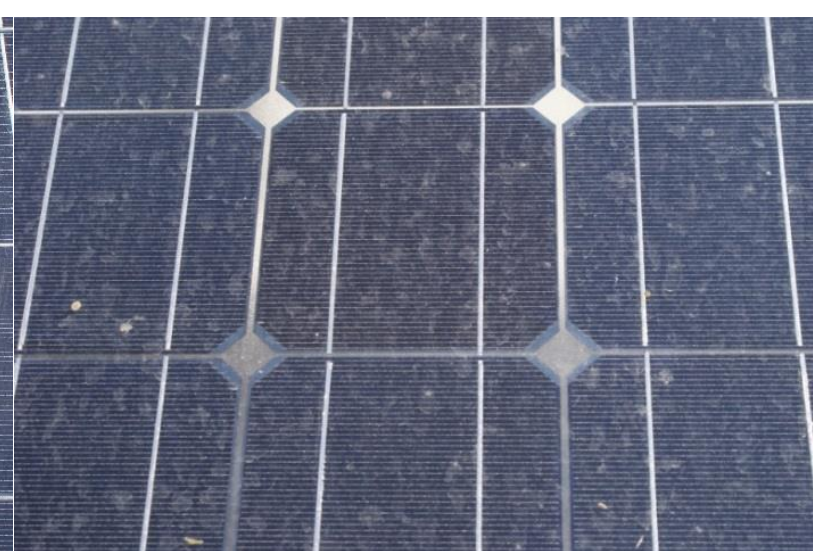

Figure 2: Image of a dusty Panel 


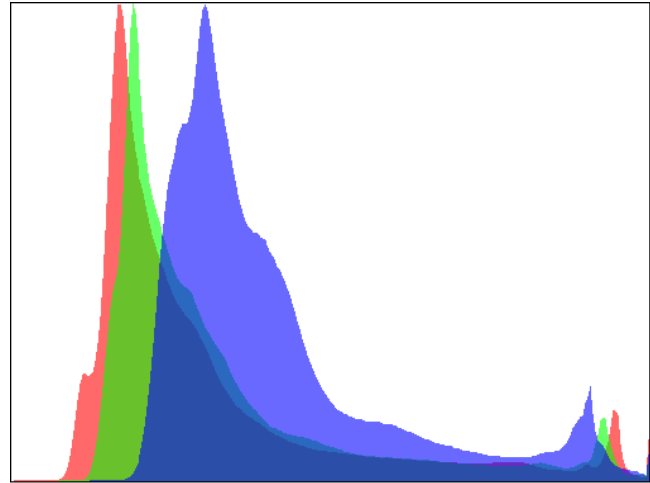

(a) Clean Panel

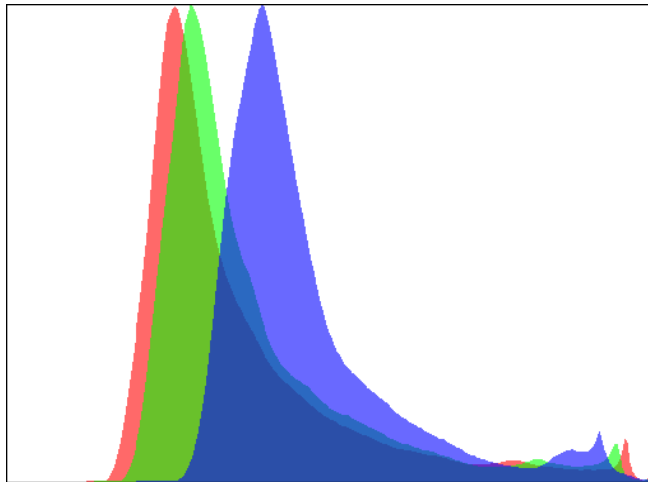

(b) Dusty Panel

Figure 3: Probability density function for the R, G, B channels, Observe the shift of the red, green, and blue modes.

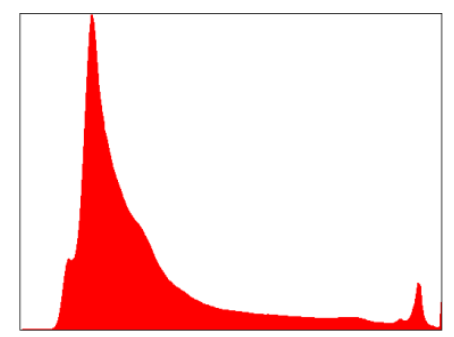

(a) Clean, Red

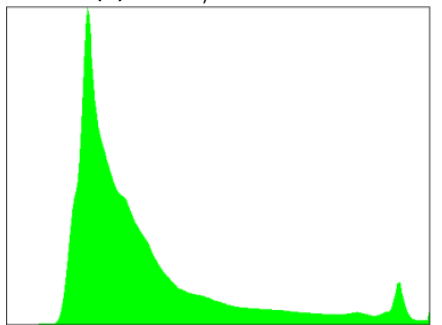

(c) Clean, Green

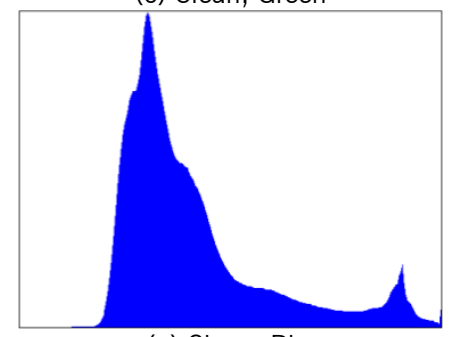

(e) Clean, Blue

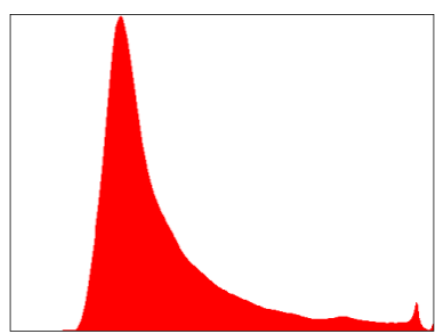

(b) Dusty, Red

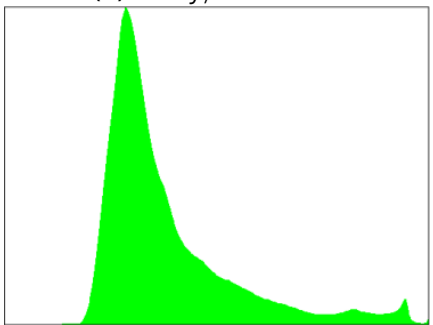

(d) Dusty, Green

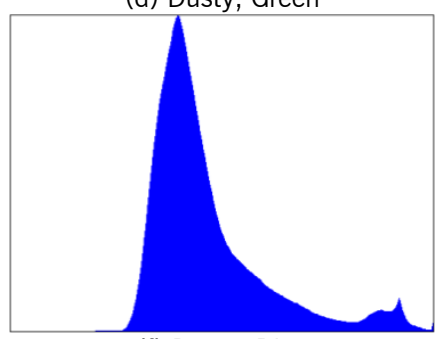

(f) Dusty, Blue

Figure 4: Probability density function for the R, G, B channels

The point $x_{m}=(\alpha-1) \beta$ is the global maximum for $f(x)$, thus it is the mode of the density function. It is very easy to prove that the mean or expected value of a gamma is $\mu=\alpha \beta$. Given a panel image we can use the pixels to extract the values of the red, green, and blue channels. For each one of these channels we can estimate the mean, and the maximum mode. The mean is the average of all the values for that channel, and the maximum mode of course is the point between 0 and 255 for which most of the values of that channel for the picture that the channel belongs to, are equal to. Let the sample mean for the arbitrary channel be denoted by $\bar{x}$ and let the estimate of the maximum mode be denoted by $\hat{x}$. Then the parameters $\alpha, \beta$, of the gamma can be estimated by setting:

$$
\bar{x}=\alpha \beta
$$

and 


$$
\widehat{x}=(\alpha-1) \beta
$$

From the above two equations we obtain

$$
\bar{x}-\beta=\hat{x}
$$

which implies that:

$$
\widehat{\beta}=\bar{x}-\hat{x}
$$

From equation III.6 therefore we obtain that

$$
\widehat{\alpha}=\frac{\bar{x}}{\bar{x}-\hat{x}}
$$

QED.

Formulas III.9 and III.10 give us estimates of the parameters $\alpha$ and $\beta$ which they define the gamma density function for the channel based on the channel data. We took the picture of each one of the fifty panels when they were dusty, then we cleaned them and we took their picture again. Thus we obtained 50 clean panel pictures and fifty dusty under the same conditions. For each one of the pictures we computed the probability density function of the red, green, and blue, channels. As we graphed each one of the probability density functions the $x$-axis denotes all the possible values a color-channel can have. Thus the values for each one of the three channels vary between 0-255. The value in the $x$-axis for which the experimental density function obtains its global maximum is the mode. For the clean pictures we obtained 50 mode data for the red channel, 50 mode data for the green, and $\mathbf{5 0}$ mode data for the blue. In each one of the pictures the mode for the blue is over 20 units higher than the mode for the green and the mode for the green is about 4 units higher than the mode for red. As the panels get dusty then the modes are shifted to higher values, and the electric energy of the panel is reduced. There is a tradeoff between the energy needed for the vacuum to clean the panel and the energy gained. There is a cutoff point beyond which not cleaning the panel significant energy is lost and therefore we need to detect that the panel has reached that cutoff point and trigger the mechanism automatically to clean the panel. The classification theory that enables us to make this decision is based on the following statistics. Consider the 50 clean panels. Let $\left(r_{m 1}, r_{m 2}, \ldots, r_{m 50}\right),\left(g_{m 1}, g_{m 2}, \ldots, g_{m 50}\right)$ and $\left(b_{m 1}, b_{m 2}, \ldots, b_{m 50}\right)$, be the 50 computed mode values for each one of the clean channels. Let $\bar{r}_{m}, \bar{g}_{m}, \bar{b}_{m}$, denote the average of the red-modes, green-modes, and blue-modes respectively. Then according to the central limit theorem the probability distribution function, of the vector $\bar{r}_{m}, \bar{g}_{m}, \bar{b}_{m}$ approaches to a trivariate normal. The random vectors $\left(r_{m 1}, g_{m 1}, b_{m 1}\right),\left(r_{m 2}, g_{m 2}, b_{m 2}\right), \ldots,\left(r_{m 50}, g_{m 50}, b_{m 50}\right)$, are independent since each panel is autonomous and independent of the other panels, and identically distributed, with a mean vector $\underline{\mu}^{\prime}=\left(\mu_{r m}, \mu_{g m}, \mu_{b m}\right)$, where the $\underline{\mu}^{\prime}$ signifies the transpose vector, and variance- covariance matrix

$$
\Sigma=\left[\begin{array}{ccc}
\sigma_{r m}^{2} & \sigma_{r m g m} & \sigma_{r m b m} \\
\sigma_{r m g m} & \sigma_{g m}^{2} & \sigma_{g m b m} \\
\sigma_{r m b m} & \sigma_{g m b m} & \sigma_{b m}^{2}
\end{array}\right]
$$

The expected value $E\left(\bar{r}_{m}, \bar{g}_{m}, \bar{b}_{m}\right)=\underline{\mu}^{\prime}=\left(\mu_{r m}, \mu_{g m}, \mu_{b m}\right)$, and the variance covariance matrix of the vector $\bar{r}_{m}, \bar{g}_{m}, \bar{b}_{m}$ is $\frac{\Sigma}{50}$. In general if $\mathrm{n}$-pictures are used to compute the vector $\bar{r}_{m}, \bar{g}_{m}, \bar{b}_{m}$, then the variance-covariance vector of $\bar{r}_{m}, \bar{g}_{m}, \bar{b}_{m}$, would be $\frac{\Sigma}{n}$. The vector $\underline{\mu}^{\prime}=$ $\left(\mu_{r m}, \mu_{g m}, \mu_{b m}\right)$ is estimated from the data. Thus if a sample of $\mathrm{n}$ clean panels is used and we denote the trivare vector of the mode of the $\mathrm{i}^{\text {th }}$ panel as $x_{m i}=\left(r_{m i}, g_{m i}, b_{m i}\right)$ then 


$$
\bar{x}_{m}=\frac{x_{m 1}+x_{m 2} \ldots+x_{m n}}{n}
$$

And the variance covariance matrix $\Sigma$ can be estimated from the data as follows:

$$
\widehat{\Sigma}=\frac{1}{n-1} \sum_{i=1}^{n}\left(x_{m i}-\bar{x}_{m}\right)\left(x_{m i}-\bar{x}_{m}\right)^{\prime}
$$

Theorem 2 creates the criterion for classifying a panel as being clean or needing cleaning.

Theorem 2. Let $x$ denote the vector of $r, g, b$, modes of a new panel and $\frac{n}{n+1}\left(x-\bar{x}_{m}\right)^{\prime} \hat{\Sigma}^{-1}\left(x-\bar{x}_{m}\right)$ then $\frac{n-3}{3(n-1)} t^{2} \sim F_{3, n-3}$ is an $\mathrm{F}$ distribution with parameters $3, \mathrm{n}-3$. If $x^{\prime} \hat{\Sigma}^{-1} x<\bar{x}_{m}{ }^{\prime} \hat{\Sigma}^{-1} \bar{x}_{m}+\frac{3\left(n^{2}-1\right)}{n(n-3)} F_{3, n-3, \varsigma}$, then the panel is classified as clean, otherwise as needed to be cleaned. $F_{3, n-3, \zeta}$ in the classifier formula is the F-distribution value with parameters 3 and $n-3$, and level of significance $\varsigma$.

\section{Proof}

Since $\mathrm{x}$ is the vector of $\mathrm{r}, \mathrm{g}, \mathrm{b}$ modes of a new panel, is independent of the $\mathrm{n}$ clean panels already used to computer $\bar{x}_{m}$. The

$$
E\left(x-\bar{x}_{m}\right)=E\left((x-\mu)-\left(\bar{x}_{m}-\mu\right)\right)=E(x-\mu)-E\left(\bar{x}_{m}-\mu\right)=0
$$

and the variance covariance matrix of the vector $x-\bar{x}_{m}$ is

$E\left(x-\bar{x}_{m}\right)\left(x-\bar{x}_{m}\right)^{\prime}=E\left[(x-\mu)-\left(\bar{x}_{m}-\mu\right)\right]\left[(x-\mu)-\left(\bar{x}_{m}-\mu\right)\right]^{\prime}=E(x-\mu)(x-\mu)^{\prime}+E\left(\bar{x}_{m}-\right.$ $\mu)\left(\bar{x}_{m}-\mu\right)^{\prime}-E(x-\mu)\left(\bar{x}_{m}-\mu\right)^{\prime}-E(x-\mu)^{\prime}\left(\bar{x}_{m}-\mu\right)=\Sigma+\frac{1}{\mathrm{n}} \Sigma-0-0=\left(1+\frac{1}{n}\right) \Sigma$

Notice that

$$
(x-\mu), \text { and }\left(\bar{x}_{m}-\mu\right)^{\prime}
$$

are statistically independent therefore

$$
E(x-\mu)\left(\bar{x}_{m}-\mu\right)^{\prime}=0
$$

For the same reason

$$
E(x-\mu)^{\prime}\left(\bar{x}_{m}-\mu\right)=0
$$

an estimate of $\Sigma$ is

$$
\widehat{\Sigma}=\frac{1}{n-1} \sum_{i=1}^{n}\left(x_{m i}-\bar{x}_{m}\right)\left(x_{m i}-\bar{x}_{m}\right)^{\prime}
$$

from this we infer that

$$
t^{2}=\frac{n}{n+1}\left(x-\bar{x}_{m}\right)^{\prime} \hat{\Sigma}^{-1}\left(x-\bar{x}_{m}\right) \sim T_{3, n-1}^{2}
$$

and therefore 


$$
\frac{n-3}{3(n-1)} t^{2} \sim F_{3, n-3}
$$

If $\varsigma$ is the level of significance chosen then the probability that

$$
\frac{n-3}{3(n-1)} \frac{n}{n+1}\left(x-\bar{x}_{m}\right)^{\prime} \hat{\Sigma}^{-1}\left(x-\bar{x}_{m}\right)<F_{3, n-3, \varsigma}
$$

is (1- $\varsigma)$, where $\varsigma$ is the level of significance and is selected to be less than or equal to 0.05 .

From the above equation we obtain

$$
\left(x-\bar{x}_{m}\right)^{\prime} \hat{\Sigma}^{-1}\left(x-\bar{x}_{m}\right)<\frac{3\left(n^{2}-1\right)}{n(n-3)} F_{3, n-3, \varsigma}
$$

or

$$
x^{\prime} \hat{\Sigma}^{-1} x<\bar{x}_{m}^{\prime} \hat{\Sigma}^{-1} \bar{x}_{m} \frac{3\left(n^{2}-1\right)}{n(n-3)} F_{3, n-3, \varsigma}
$$

The right hand side of the above inequality is computed from the clean data after the sampling and formulation of the classifier. Every time we need to classify a new panel then we compute its vector $\mathrm{x}$ of $\mathrm{r}, \mathrm{g}, \mathrm{b}$, modes and after computing the left hand side $x^{\prime} \hat{\Sigma}^{-1} x$ of the above equation we compare it with the right hand side. If it satisfies the above inequality then we classify it as clean otherwise we trigger the cleaning mechanism to clean it.

\section{Conclusion}

Solar panels in the arid climates produce a great deal of energy because the sun shines either throughout the year, or close to 350 days of the year. Sandstorms, small animals, and birds, depositing their droppings are responsible for the reduction of the energy produced by the panels. Here we introduced an algorithm that takes as input the panel picture and decides if the panel is clean or needs cleaning. This project is relatively new. The algorithm we described in this paper is still being tested. So far our classification algorithm seems to be working. As the panels get dusty or subjected to bird droppings but they do not have enough deposits to pass our cut off point we see that the energy drop is within the normal fluctuation energy of clean panels. As they get dustier to the point that the inequality III.21 is not being satisfied then we see that there is enough drop in energy that by investing some energy to clean the panel will increase the energy output enough so that we can recover the energy invested by the panel within a few hours and have an energy gain until the next cleaning is required. The mean time between cleaning could be several months or less than a day depending on the weather conditions, the day of the month, the month of the year, and even the sun spot periodicity.

\section{ACKNOWLEDGMENT}

The authors would like to thank Dr. R. F. Boehm for the generous access he has provided to the solar panels at his UNLV lab, and his continuing support. Many thanks to the NSF for their support and funding of this project.

\section{REFERENCES}

[1]. C. E. Bohren and A. B. Fraser, "Colors of the Sky," The Physics Teacher, no. 5, 1985, pp. 269-272.

[2]. H. C. V. D. Hulst, "Light Scattering by Small Particles," John Wiley and Sons Inc., New York, 1957. 
E. A. Yfantis \& A. Fayed; A Camera System For Detecting Dust And Other Deposits On Solar Panels, Advances in Image and Video Processing, Volume 2 No 5, Oct (2014); pp: 1-10

[3]. P. E. Haralabidis, and C. Pilinis, "Skylight Color Shifts due to Variations of Urban-Industrial Aerosol Properties: Observer Color Difference Sensitivity Compared to a Digital Camera," Aerosol Science and Technology, vol. 8, no 42, pp. 658-673, 2008.

[4]. E. Zamora Ramos, "Using Image Processing Techniques To Estimate The air Quality," Journal of Mcnair Scholars Institute, $6^{\text {th }}$ Edition, pp. 189-194.

[5]. S.-C Tsay, G. L. Stephens and T. J. Greenwald,, "An Investigation of aerosol Microstructure on Visual air Quality," atmospheric environment, vol. 25A, no 5/6, pp. 1039-1053, 1991.

[6]. R. v. Hogg and A. T. Craig, "Introduction to mathematical Statistics,", Fourth Edition, Macmillan Publishing Co., pp. 1-438, 1978.

[7]. S. Theodoridis, K. Koutroumbas, "Pattern recognition," fourth edition, Academic Press, pp. 1-961. 